\title{
Location-Specific ASPECTS Paradigm in Acute Ischemic Stroke: A Systematic Review and Meta-Analysis
}

\author{
(D) S.M. Seyedsaadat, (D) A.A. Neuhaus, (D).M. Pederson, (D)W. Brinjikji, (D)A.A. Rabinstein, and (D)D.F. Kallmes
}

\begin{abstract}
BACKGROUND: Weighting neuroimaging findings based on eloquence can improve the predictive value of ASPECTS, possibly aiding in informed treatment decisions for acute ischemic stroke.
\end{abstract}

PURPOSE: Our aim was to study the contribution of region-specific ASPECTS infarction to acute ischemic stroke outcomes.

DATA SOURCES: We searched MEDLINE and EMBASE for reports on ASPECTS in patients with acute ischemic stroke from 2000 to March 2019.

STUDY SELECTION: Two investigators independently reviewed articles and extracted data. Three-month poor functional outcome defined as $m R S>2$ was the primary end point.

DATA ANALYSIS: A random-effects meta-analysis was performed to compare the association between infarct and mRS $>2$ among ASPECTS regions. Subanalyses included the following: laterality of stroke (left/right), imaging technique (NCCT or advanced imaging with DWI, CTP, or CTA), and interventional technique (IV-tPA/conservative management or mechanical thrombectomy).

DATA SYNTHESIS: M6 infarct was most associated with poor functional outcome (OR = 3.26; 95\% Cl, 2.21-4.80; $P<.001)$. Pair-wise comparisons of ASPECTS regions regarding the association between infarct and $m R S>2$ were not significant, with the exception of $M 6$ versus lentiform $(P=.009)$. However, pair-wise comparisons among ASPECTS regions were not significant among subgroup analyses.

LIMITATIONS: Limitations were the heterogeneity of time points, neuroimaging modalities, and interventional techniques; limited studies for inclusion; publication bias among some comparisons; and the retrospective nature of included studies.

CONCLUSIONS: Our study indicated an unequal impact of some ASPECTS subregions in predicting outcomes of patients with acute ischemic stroke. Stroke laterality, imaging technique, and interventional technique subgroup analyses showed no differences among ASPECTS regions in predicting outcome. Investigation in larger cohorts is required to assess the association of ASPECTS with acute ischemic stroke outcome.

ABBREVIATIONS: AIS = acute ischemic stroke; $\mathrm{M} 1=$ anterior inferior frontal lobe; $\mathrm{M} 2=$ temporal lobe; $\mathrm{M} 3=$ inferior parietal and posterior temporal lobe; M4 = anterior superior frontal lobe; M5 = precentral and superior frontal lobe; M6 = superior parietal lobe; MT = mechanical thrombectomy; PRISMA = Preferred Reporting Items for Systematic reviews and Meta-Analysis

D

espite advances in endovascular therapy, patients with acute ischemic stroke (AIS) often have disabilities following treatment,

Received January 21, 2020; accepted after revision July 7.

From the Departments of Radiology (S.M.S., W.B., D.F.K.), and Neurology (A.A.R.), Mayo Clinic, Rochester, Minnesota; Medical School (A.A.N.), University of Oxford, Oxford, England; Department of Veterinary and Biomedical Sciences (J.M.P.), University of Minnesota, Minneapolis, Minnesota; and Superior Medical Experts (). M.P.), St. Paul, Minnesota.

Please address correspondence to John M. Pederson, MD, 1430 Avon St N, Saint Paul, MN 55117; e-mail: jpederson@supedit.com

Indicates article with supplemental on-line tables.

Indicates article with supplemental on-line photo.

http://dx.doi.org/10.3174/ajnr.A6847 indicating the importance of continued refinement in patient-selection criteria. ${ }^{1}$ Patient selection for reperfusion therapy is based on brain neuroimaging and time windows; however, standardized guidelines for establishing strict inclusion and exclusion criteria have not been established. ${ }^{2,3}$ Following recent findings from multiple landmark randomized clinical trials that established the advantages of endovascular therapy over medical therapy, the American Heart Association Guidelines recommended ASPECTS $\geq 6$ as a cutoff point for treatment. ${ }^{4-9}$ However, recent studies have indicated that not only would most patients with baseline ASPECTS $\geq 6$ achieve good functional outcome but also that up to $42 \%$ of successfully recanalized patients with low baseline ASPECTS (ASPECTS 
55) can achieve good functional outcome. ${ }^{10}$ Conversely, patients with relatively high ASPECTS scores may have poor outcomes even after recanalization, highlighting the limited utility of this prognostic tool. These limitations may lead to withholding the criterion standard treatment from patients eligible for reperfusion therapy or may result in treating patients in whom endovascular therapy is unwarranted.

The occlusion site and the resulting ischemic regions are the most important predictors of anterior circulation AIS outcome. ${ }^{11,12}$ The limited predictive value of ASPECTS might be explained by the lack of consideration of functional eloquence across the 10 prespecified brain regions included in the scale. For example, the lentiform nucleus on the nondominant side and M2 on the dominant side contribute equally to the composite ASPECTS, though their functional roles are distinct. By addressing this limitation, better evidence-based clinical decision-making for patients with AIS could be achieved. Indeed, a growing number of studies are supporting the consideration of infarct location and stroke laterality for stroke-outcome prediction in addition to the total DWI or CT ASPECTS score. ${ }^{12-15}$ Consequently, we performed this systematic review and meta-analysis to study the relationship between infarct topography on individual ASPECTS regions and AIS outcomes.

\section{MATERIALS AND METHODS Literature Search}

This study is reported according to the Preferred Reporting Items for Systematic reviews and Meta-Analysis (PRISMA) guidelines. We searched Ovid MEDLINE, Epub Ahead of Print, In-Process, and Other Non-Indexed Citations, Ovid EMBASE, Scopus EMBASE, PubMed, and Google Scholar using predefined keywords and synonyms for studies reporting ASPECTS scores in patients with AIS from 2000 (when ASPECTS was published) to the end of March 2019. An experienced librarian designed and performed the search strategy with input from the principal and senior investigators. We searched for key terms including "Alberta Stroke Program Early CT Score," "ASPECTS," and "ischemic stroke." We also reviewed the reference lists of potentially included articles searching for additional relevant studies.

\section{Study Eligibility}

Human studies that reported 90-day clinical outcomes related to each individual ASPECTS score were included. We excluded studies with the following criteria: 1) case reports; 2) review articles; 3 ) technical notes, editorial comments, or letters; 4) non-English language; 5) conference abstracts with no separated results for each ASPECTS region; and 6) nonrelevant to the study topic. The primary outcomes of interest were associations between poor neurologic outcomes ( $\mathrm{mRS}>2$ ) and deficits in each ASPECTS region.

\section{Data Extraction, Baseline Variables, and Outcome}

Studies that were included on the basis of titles and abstracts were screened by a postdoctoral research fellow experienced in neurointerventional medicine. Subsequently, 2 medical doctors with 10 and 3 years of research experience in neuroradiology independently extracted and verified data from the full text of eligible articles. Disagreements were resolved by consensus or consulting with a senior coauthor. The following baseline information was collected from each study: title, first author, year of publication, patient demographics, study design, number of patients, comorbidities, baseline NIHSS score, stroke laterality, ASPECTS, imaging technique, imaging time, and type of treatment. The primary end point of this study was poor functional outcome, defined as a mRS $>2$ at 3 months.

\section{Risk of Bias Assessment}

The Quality in Prognosis Studies (QUIPS; https://abstracts. cochrane.org/2017-global-evidence-summit/systematic-reviewsprognostic-studies-ii-assessing-bias-studies) tool was used to assess the risk of bias of the included studies. ${ }^{16}$ Using this risk-ofbias tool, we assessed each study on the basis of 6 criteria under 3 main categories, including 1) selection of study groups; 2) comparability of the study groups; and 3) ascertainment of the outcome. The risk of bias was classed as high, moderate, or low.

Studies with an unclear risk of bias were considered as moderate risk. The full risk-of-bias assessment can be found in On-line Table 1.

\section{Statistical Analysis}

ORs with 95\% CIs were estimated for each study outcome. ORs were pooled across studies using a random-effects meta-analysis. ${ }^{17}$ Heterogeneity across studies that was not attributable to chance was assessed using the $\mathrm{I}^{2}$ statistic, with $\mathrm{I}^{2}>50 \%$ suggesting substantial heterogeneity. ${ }^{18}$ Publication bias across included studies was explored by funnel plots and testing their symmetry by using the Egger regression. ${ }^{19}$ Subgroup analyses were categorized by follow-up time, type of neuroimaging, stroke laterality, and mechanical thrombectomy (MT) versus tPA/conservative management subgroups. Brain topography maps indicating correlations between infarct in individual ASPECTS regions and poor neurologic outcome $(\mathrm{mRS}>2)$ are represented as $\beta$-coefficients from pooled ORs that were normalized to a maximum value of 1.0; thus, the ASPECTS region most strongly associated with poor neurologic outcome was assigned a value of 1.0 , and the remaining regions were assigned values relative to the maximum and according to their respective $\beta$-coefficients. The analysis was performed using Excel for Office 365 (Version 1909; Microsoft) for basic summary statistics and data organization and Comprehensive MetaAnalysis, Version 3.3 (Biostat) for calculating odds ratios.

\section{RESULTS}

\section{Literature Search}

Among 1035 search results, 923 articles were duplicates, nonoriginal, or irrelevant to the aim of our study and were thus excluded after reviewing the title and abstracts alone. Of the remaining 112 eligible articles for full-text review, 103 were excluded because they did not provide the outcome for each individual ASPECTS region. Ultimately, 9 articles with 2249 unique patients were included in the random-effects meta-analysis. ${ }^{12-15,20-24}$ Figure 1 shows the PRISMA flow diagram of the process of screening and selection of the eligible studies.

\section{Study and Patient Characteristics}

Of the 9 included studies (On-line Table 1), 5 studies were multicenter and 4 were single-center. Three studies were retrospective, and 6 were prospectively designed. The mean age of patients was 67 years, with a slight male preponderance (50.5\%). Six studies 
evaluated patients using NCCT, while 2 used DWI and 1 used CTP for scoring ASPECTS. Six studies used admission neuroimaging reports, while 3 used images within 12-72 hours after stroke onset. Baseline characteristics of patients and studies are summarized in On-line Table 1.

\section{Outcome}

Region-Specific ASPECTS and Infarct Distribution. Overall, infarction was most frequent in the insula (52\%) and lentiform nucleus $(51.5 \%)$ and least frequent in the internal capsule (23.6\%) and
M3 (27.9\%). A summary of the overall distribution of infarction within ASPECTS regions is shown in On-line Table 2.

Publication Bias and Study Heterogeneity. The risk of bias was not significant $(P$ value $>.05)$ for pooled analyses of the M1-M6 and the caudate brain regions; however, the Egger regression showed significant publication bias regarding pooled assessments of the internal capsule, lentiform nucleus, and insula regions. Heterogeneity with regards to poor functional outcome among was low to moderate for all ASPECTS regions $\left(\mathrm{I}^{2}<50 \%\right.$; Table).

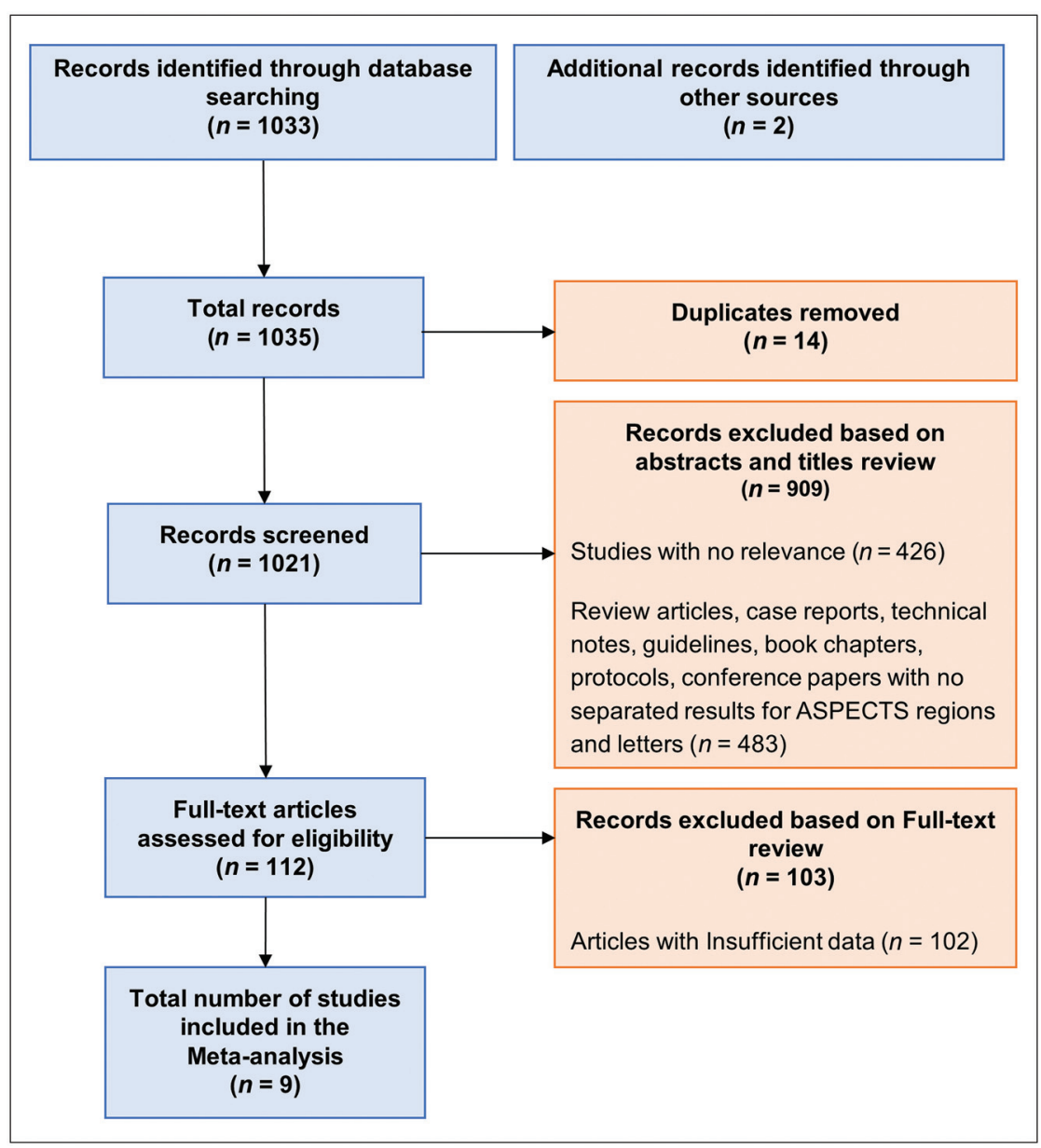

FIG 1. Flow diagram depicting the literature review, search strategy, and selection process using the PRISMA guidelines.
Region-Specific ASPECTS and Outcome. Figure 2 shows a heat map that indicates the weight of each individual ASPECTS region infarct in predicting outcome, based on the $\beta$-coefficients from pooled ORs from included studies. A forest plot of pooled results for the effect of per-region ASPECTS on outcome is provided in Fig 3 (see the On-line Figure for forest plots showing both study-level and pooled results from each ASPECTS region). Across different time points (baseline versus follow-up) and among different modalities (NCCT, CTP, MR imaging), the strongest contribution to long-term outcome was from infarction of M6 (OR = 3.26; 95\% CI, 2.21-4.80; $P<.001)$. This was followed by M3 (OR $=2.42 ; 95 \%$ CI, 1.45-4.04; $P<.001)$ and M2 (OR = 2.40; 95\% CI, 1.56-3.66; $P<.001$ ). ORs from cortical areas were higher than those of subcortical regions in all cases. Overall, caudate $(\mathrm{OR}=1.48 ; 95 \% \mathrm{CI}$, 0.94-2.33; $P=.092)$ and lentiform (OR $=1.41 ; 95 \%$ CI, 0.98-2.02; $P=.065$ ) infarcts had the weakest association with poor outcome. Pair-wise comparisons between individual ASPECTS regions regarding the association between infarct and poor neurologic outcome were not significant, with the exception of M6 versus the lentiform nucleus $(P=.009)$.

\section{$I^{2}$ Statistics and the Egger weighted regression statistics}

\begin{tabular}{lrlrl}
\hline Region & $\mathbf{I}^{\mathbf{2}}$ & \multicolumn{1}{c}{$\mathbf{I}^{\mathbf{2}}$ Interpretation } & Egger Intercept & Interpretation \\
\hline M1 & $0.00 \%$ & Low heterogeneity & $0.308(P=.372)$ & Publication bias not significant \\
M2 & $19.56 \%$ & Low heterogeneity & $0.532(P=.309)$ & Publication bias not significant \\
M3 & $32.84 \%$ & Moderate heterogeneity & $-0.026(P=.491)$ & Publication bias not significant \\
M4 & $11.44 \%$ & Low heterogeneity & $-0.207(P=.414)$ & Publication bias not significant \\
M5 & $2.85 \%$ & Low heterogeneity & $0.211(P=.373)$ & Publication bias not significant \\
M6 & $18.21 \%$ & Low heterogeneity & $-0.066(P=.468)$ & Publication bias not significant \\
C & $17.36 \%$ & Low heterogeneity & $-1.290(P=.085)$ & Publication bias not significant \\
IC & $5.89 \%$ & Low heterogeneity & $-1.372(P=.031)$ & Significant publication bias \\
L & $14.91 \%$ & Low heterogeneity & $-1.406(P=.018)$ & Significant publication bias \\
I & $40.55 \%$ & Moderate heterogeneity & $-1.670(P=.023)$ & Significant publication bias \\
\hline
\end{tabular}

Note:-C indicates caudate; IC, internal capsule; L, lentiform; I, insula. 


\section{Sensitivity Analysis}

NCCT versus Advanced Imaging (DWI, CTP, and CTA). Regarding the weights of individual ASPECTS regions in predicting outcome when ischemic, M6 (OR = 3.29; 95\% CI, 1.68-6.45; $P<.001)$ and M3 (OR $=2.75 ; 95 \% \mathrm{CI}, 1.19-6.39 ; P=.001)$ infarctions on NCCT were stronger predictors of poor functional outcome than other ASPECTS regions (Fig 4). For NCCT ASPECTS, the caudate nucleus and insula had the weakest association with poor outcome. For DWI ASPECTS studies, M6 (OR 3.38; 95\% CI, 2.07-5.50; $P<.001$ ) and M4 (OR 3.02; 95\% CI, 1.82-5.00; $P<.001)$ were more strongly associated with poor functional outcome than other regions (Fig 5). On DWI ASPECTS, lentiform and M3 infarctions had the weakest contribution to long-term function. Pair-wise comparisons among individual ASPECTS regions regarding the association between infarct and poor neurologic outcome were not significant in all cases when

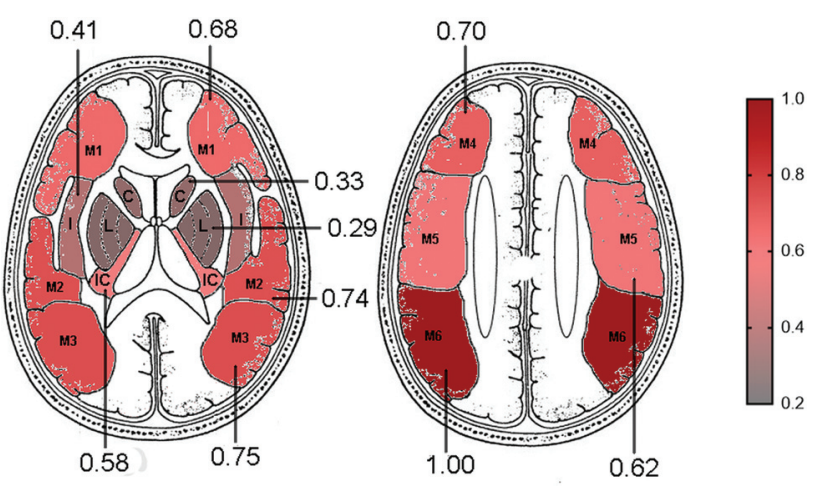

FIG 2. Topography and outcome brain map generated using the $\beta$-coefficients from pooled ORs for each region based on the random-effects model, indicating the relative weight of each ASPECTS region to $M 6$, which was determined to be the region with greatest significant association with poor outcomes. The ASPECTS region most strongly associated with poor neurologic outcome was assigned a value of 1.0, and the remaining regions were assigned values relative to the maxima and according to their respective $\beta$-coefficients. Reprinted with permission from Barber et al. ${ }^{34} \mathrm{C}$ indicates caudate nucleus; L, lentiform nucleus; IC, internal capsule; I, insula. categorizing by imaging technique. The results of pooled analyses from studies measuring region-specific infarcts in ASPECTS locations via CT or MR imaging and associated clinical outcome can be found in On-line Tables 3 and 4, respectively.

Laterality of Stroke. The pooled analysis of studies that evaluated infarcts by laterality showed that only M1, M5, and M6 were significantly associated with poor outcome on the right side (On-line Table 5). A brain topography map indicating the association between infarct in individual ASPECTS regions for right-sided strokes is presented in Fig 6. On the left side, the pooled analysis of observed data demonstrated that only the M1, M2, M4, M5, M6, and the lentiform nucleus were significantly associated with poor outcome (On-line Table 5). A brain topography map indicating the association among infarcts in individual ASPECTS regions for leftsided strokes is also presented in Fig 6. Pair-wise comparisons among individual ASPECTS regions regarding the association between infarct and poor neurologic outcome were not significant in all cases when categorizing by laterality.

MT versus tPA/Conservative Management. The pooled analysis of 4 studies that evaluated infarcts in patients who were treated with only MT showed that M4 (OR $=2.78$; 95\% CI, $1.75-4.4 ; P<.001)$ and M6 (OR $=2.59 ; 95 \% \mathrm{CI}, 1.37-4.9 ; P=.003)$ were the strongest predictors of poor outcome (On-line Table 6). The pooled analysis of 2 studies that assessed infarcts in patients who were treated with only IV tPA/conservative management demonstrated that M6 $(\mathrm{OR}=2.88 ; 95 \% \mathrm{CI}, 1.4-5.91 ; P=.004)$ and $\mathrm{M} 3(\mathrm{OR}=2.76 ; 95 \%$ $\mathrm{CI}, 1.18-3.65 ; P=.011)$ were the strongest predictors of poor outcome (On-line Table 7).

\section{DISCUSSION}

The collective findings of the 9 studies in the current meta-analysis showed that infarcts in M6 were the strongest predictors of AIS outcome compared with other ASPECTS regions; however, these findings were only statistically significant with respect to infarcts in the lentiform nucleus. When analyzed by technique, infarction in M6 and M2 on NCCT and M6 and M4 on DWI had the greatest relative association with poor AIS outcome. In addition, infarction in M6 and M2 on the right and M5 and M4 on the left side were associated with worse functional outcome. However, pair-wise comparisons among individual ASPECTS regions regarding the association between infarct and poor neurologic outcome were not statistically significant when categorizing by imaging technique or laterality. Sub-group analyses were performed to separately evaluate the association of ASPECTS location and outcome in the MT group versus IV tPA/conservative management groups. As shown in On-line Tables 6 and 7 for MT and tPA/conservative management, there were no substantial differences for

FIG 3. Forest plots demonstrating the contribution of individual ASPECTS regions, with 90-day functional outcome based on collective data of the 9 included studies. 


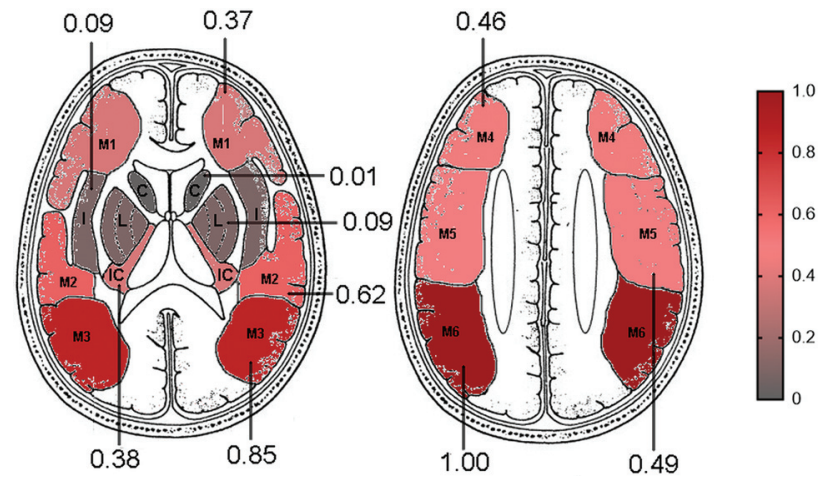

FIG 4. Topography and outcome brain map generated using the $\beta$-coefficients from pooled ORs for each region based on NCCT ASPECTS studies. Note that data shown are calculated from both hemispheres but are displayed unilaterally for clarity. The ASPECTS region most strongly associated with poor neurologic outcome was assigned a value of 1.0 , and the remaining regions were assigned values relative to the maxima and according to their respective $\beta$-coefficients. Reprinted with permission from Barber et al. ${ }^{34} \mathrm{C}$ indicates caudate nucleus; L, lentiform nucleus; IC, internal capsule; I, insula.
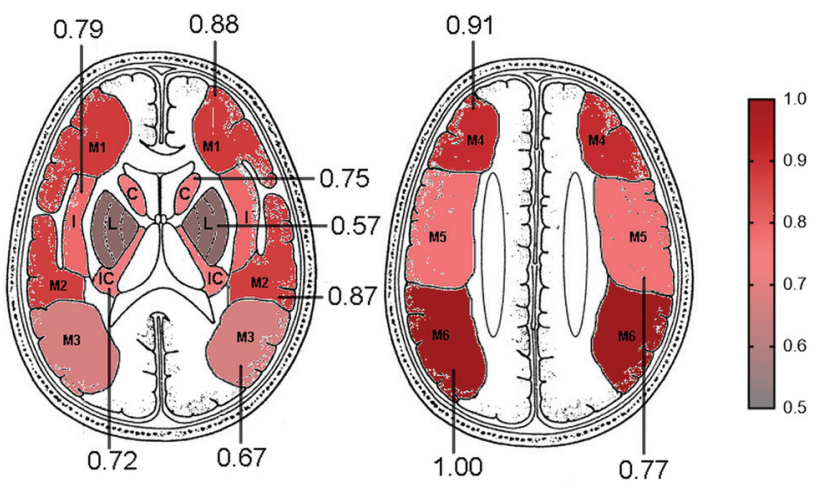

FIG 5. Topography and outcome brain map generated using the $\beta$-coefficients from pooled ORs for each region based on DWI ASPECTS studies. Note that data shown are calculated from both hemispheres but are displayed unilaterally for clarity. The ASPECTS region most strongly associated with poor neurologic outcome was assigned a value of 1.0, and the remaining regions were assigned values relative to the maxima and according to their respective $\beta$-coefficients. Reprinted with permission from Barber et al. ${ }^{34} \mathrm{C}$ indicates caudate nucleus; L, lentiform nucleus; IC, internal capsule; I, insula.

individual ASPECTS regions both within and between the subgroups.

Previous studies showed that proximal MCA occlusion is associated with worse outcome and worse recanalization than distal MCA occlusion; ${ }^{25}$ and the corona radiata, internal capsule, and insula have a high influence on 30-day functional outcome following AIS. ${ }^{26}$ In contrast, our results suggest that cortical areas may have a greater influence on long-term outcome.

In all 3 studies ${ }^{14,20,21}$ that used admission CT, the insula and lentiform nucleus were the 2 most frequently infarcted regions. However, among the 4 studies $^{12,13,15,24}$ that used advanced neuroimaging modalities, insula followed by M5 were the most frequent regions. Most interesting, among all 10 ASPECTS regions, the internal capsule (10.4\%) was the least common site of infarction on either admission or 12- to 72-hour CT or DWI.
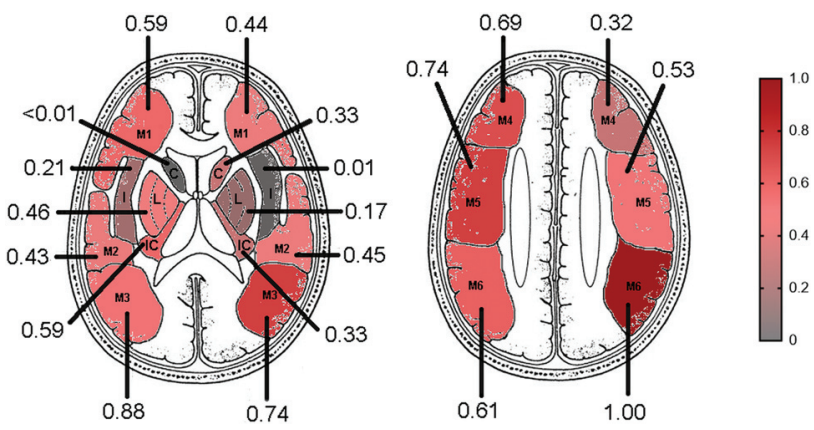

FIG 6. Topography and outcome brain map generated using the $\beta$-coefficients from pooled ORs for each region based on the laterality of the stroke. The ASPECTS region most strongly associated with poor neurologic outcome was assigned a value of 1.0, and the remaining regions were assigned values relative to the maxima and according to their respective $\beta$-coefficients. Reprinted with permission from Barber et al. ${ }^{34} \mathrm{C}$ indicates caudate nucleus; L, lentiform nucleus; IC, internal capsule; I, insula.

Although NCCT is easy-to-use, time-saving, and the most commonly accessible imaging technique for the evaluation of the extent of early ischemic change, ${ }^{4,27}$ early ischemic changes might not be readily apparent on CT. ${ }^{13}$ The lower sensitivity and interrater reliability of NCCT ASPECTS, compared with DWI ASPECTS, has led to the use of advanced neuroimaging tools with higher reliability and reproducibility, such as MR imaging and CTP in recent trials. ${ }^{23,28}$ The insula was the only region that was consistently associated with poor outcome in the 2 studies $^{22,23}$ that used advanced imaging modalities on admission. The insula is the most vulnerable region to ischemia, and its infarction is associated with delayed recovery from aphasia and paralysis. ${ }^{29,30}$ M6 was not associated with poor outcome in advanced imaging studies on admission; however, with posttreatment DWI ASPECTS, M6 infarction was significantly associated with poor outcomes. ${ }^{15,24}$ In particular, the right-hemisphere M6 was significantly associated with poor clinical outcome, perhaps due to its association with neglect syndromes. This finding has potential implications for the treatment of patients with established M6 infarction because their outcome may be comparatively poor for a given total ASPECTS value.

A few previous studies ${ }^{12,13,15,24,31}$ have evaluated the association of ischemic involvement of different ASPECTS brain regions with functional outcome of patients with AIS separately in each hemisphere using laterality as a surrogate of hemispheric dominance. While the right M6 infarction and preservation were the most associated with poor and good outcome, respectively, no ASPECTS regions distributed by laterality were significantly different in terms of ischemic involvement. There was no specific location in the left hemisphere that was consistently associated with AIS outcome in any of these 4 studies. ${ }^{12,13,15,24}$

Most interesting, despite our assumptions regarding the functional importance of the internal capsule, its infarction was significantly associated with poor outcomes in only 1 study after adjustment for other potential covariates. Furthermore, the posterior limb of the internal capsule is generally only infarcted in the setting of ICA occlusions involving the anterior choroidal artery origin, and ICA occlusions were found in only a minority of patients included in this study. ${ }^{32}$ 
Tan et $\mathrm{al}^{33}$ did not investigate infarction directly but analyzed CTA source images, defined as having equal (scoring 2), less prominent (scoring 1 ), or poor collaterals (scoring 0 ) compared with the contralateral side, resulting in a 20-point scale on the ASPECTS template. Having excluded the subcortical regions due to poor interrater reliability (see below), they found M5 collaterals to be the only significant predictor of good functional outcome. These results are supported with the poor outcome associated with M5 infarction that was reported by Haranhalli et al. ${ }^{23}$

To date, few studies have evaluated the combined effects of per-region ASPECTS infarction on the outcome of patients with AIS. While our meta-analysis did not show significant differences for most pair-wise comparisons for individual ASPECTS regions in terms of meaningful contribution to ischemic change, we suspect that this was due to limited data and a high degree of heterogeneity within the available data. Nevertheless, the results of this meta-analysis could extend the findings of previous studies with relatively small sample sizes. By elucidating the association of each ASPECTS region with long-term outcome after infarction, it could be possible to devise an adjusted ASPECTS scale that weights each region in accordance with these correlations. Such a clinical scale could potentially enhance prognostication as well as inform treatment decisions for patients with AIS. However, future investigations with large cohorts are required to assess the association of ASPECTS with AIS outcome.

One potential limitation of our meta-analysis is that previous studies have not separately reported the relationship of individual ASPECTS regions with outcome based on the recanalization rate and extension of the infarct. Therefore, we were unable to evaluate the influence of the ASPECTS location in relation to the recanalized-versus-unrecanalized vessel and extension of the infarct subgroups. Future studies are recommended to evaluate the relationship of ASPECTS location with AIS outcome in different subgroups, including recanalization status and extent of the primary infarct.

\section{CONCLUSIONS}

Our review of 9 articles provided valuable pooled data regarding the importance of location-specific infarct in each ASPECTS region for AIS prognostication. The results of previous studies included in this review article indicate an uneven distribution of infarction in different ASPECTS regions and suggest that ASPECTS regions may be unequally weighted in predicting the outcome of patients with AIS. With regard to stroke location, right M6 infarction was the strongest predictor of poor outcome; however, there was no consensus regarding the most important prediction of stroke outcome in the left hemisphere. Creating and validating eloquence-weighted ASPECTS, taking into account the different neurologic functions of each ASPECTS region, could improve the prediction of prognosis and patient selection for embolectomy.

\section{Data Sharing Statement}

Data can be made available by contacting the corresponding author via email.

Disclosures: Seyed Mohammad Seyedsaadat-RELATED: Grant: American Heart Association, Comments: I was awarded a 2-year postdoctoral research fellowship grant by American Heart Association. Ain A. Neuhaus-RELATED: Grant: Oxford University Clinical Academic Graduate School, Comments: academic grant to support travel and living expenses as a visiting scholar at the Mayo Clinic. John M. Pederson—RELATED: Consulting Fee or Honorarium: Superior Medical Experts*; Fees for Participation in Review Activities Such as Data Monitoring Boards, Statistical Analysis, Endpoint Committees, and the Like: Superior Medical Experts*; Payment for Writing or Reviewing the Manuscript: Superior Medical Experts*; Provision of Writing Assistance, Medicines, Equipment, or Administrative Support: Superior Medical Experts*; UNRELATED: Board Membership: Superior Medical Experts*; Employment: Superior Medical Experts and Marblehead Medical*; Payment for Manuscript Preparation: Superior Medical Experts*; Patents (Planned, Pending or Issued): Nested Knowledge*; Stock/Stock Options: Superior Medical Experts, Nested Knowledge.* Alejandro A. Rabinstein-UNRELATED: Royalties: Elsevier, Wolters Kluwer, Springer Publishing. David F. Kallmes-RELATED: Grant: American Heart Association, Comments: Postdoctoral Fellow Award*; UNRELATED: Grants/Grants Pending: Medtronic, MicroVention, NeuroSigma, Neurogami Medical, Inc., Insera Therapeutics*; Patents (Planned, Pending or Issued): Patents related to balloon-guided technology*; Stock/Stock Options: Marblehead Medical, Superior Medical Experts. *Money paid to the institution.

\section{REFERENCES}

1. Spiotta AM, Vargas J, Hawk H, et al. Impact of the ASPECT scores and distribution on outcome among patients undergoing thrombectomy for acute ischemic stroke. J Neurointerv Surg 2015;7:55158 CrossRef Medline

2. Pfaff J, Herweh C, Pham M, et al. Mechanical thrombectomy in patients with acute ischemic stroke and lower NIHSS scores: recanalization rates, periprocedural complications, and clinical outcome. AJNR Am J Neuroradiol 2016;37:2066-71 CrossRef Medline

3. Ryu CW, Shin HS, Park S, et al. Alberta Stroke Program Early CT Score in the prognostication after endovascular treatment for ischemic stroke: a meta-analysis. Neurointervention 2017;12:20-30 CrossRef Medline

4. Powers WJ, Derdeyn CP, Biller J, et al; American Heart Association Stroke Council. 2015 American Heart Association/American Stroke Association Focused Update of the 2013 Guidelines for the Early Management of Patients with Acute Ischemic Stroke Regarding Endovascular Treatment: a Guideline for Healthcare Professionals From the American Heart Association/American Stroke Association. Stroke 2015;46:3020-35 CrossRef Medline

5. Campbell BC, Mitchell PJ, Kleinig TJ, et al; EXTEND-IA Investigators. Endovascular therapy for ischemic stroke with perfusion-imaging selection. N Engl J Med 2015;372:1009-18 CrossRef Medline

6. Berkhemer OA, Fransen PS, Beumer D, et al; MR CLEAN Investigators. $A$ randomized trial of intraarterial treatment for acute ischemic stroke. N Engl J Med 2015;372:11-20 CrossRef Medline

7. Goyal M, Demchuk AM, Menon BK, et al; ESCAPE Trial Investigators. Randomized assessment of rapid endovascular treatment of ischemic stroke. N Engl J Med 2015;372:1019-30 CrossRef Medline

8. Jovin TG, Chamorro A, Cobo E, et al. REVASCAT Trial Investigators. Thrombectomy within $\mathbf{8} \mathbf{~ h r}$ after symptom onset in ischemic stroke. N Engl J Med 2015;372:2296-2306 CrossRef Medline

9. Saver JL, Goyal M, Bonafe A, et al; SWIFT PRIME Investigators. Stentretriever thrombectomy after intravenous t-PA vs. t-PA alone in stroke. N Engl J Med 2015;372:2285-95 CrossRef Medline

10. Broocks G, Hanning U, Flottmann F, et al. Clinical benefit of thrombectomy in stroke patients with low ASPECTS is mediated by oedema reduction. Brain 2019;142:1399-1407 CrossRef Medline

11. Sillanpaa N, Saarinen JT, Rusanen H, et al. Location of the clot and outcome of perfusion defects in acute anterior circulation stroke treated with intravenous thrombolysis. AJNR Am J Neuroradiol 2013;34:10006 CrossRef Medline

12. Khan M, Baird GL, Goddeau RP Jr, et al. Alberta Stroke Program Early CT Score infarct location predicts outcome following M2 occlusion. Front Neurol 2017;8:98 CrossRef Medline

13. Sheth SA, Malhotra K, Liebeskind DS, et al. Regional contributions to poststroke disability in endovascular therapy. Interv Neurol 2018;7:533-43 CrossRef Medline 
14. Phan TG, Demchuk A, Srikanth V, et al. Proof of concept study: relating infarct location to stroke disability in the NINDS rt-PA trial. Cerebrovasc Dis 2013;35:560-65 CrossRef Medline

15. Rangaraju S, Streib C, Aghaebrahim A, et al. Relationship between lesion topology and clinical outcome in anterior circulation large vessel occlusions. Stroke 2015;46:1787-92 CrossRef Medline

16. Hayden JA, van der Windt DA, Cartwright JL, et al. Assessing bias in studies of prognostic factors. Ann Intern Med 2013;158:280-86 CrossRef Medline

17. DerSimonian R, Laird N. Meta-analysis in clinical trials. Control Clin Trials 1986;7:177-88 CrossRef

18. Higgins JP, Thompson SG, Deeks JJ, et al. Measuring inconsistency in meta-analyses. Br Med J 2003;327:557-60 CrossRef Medline

19. Egger M, Davey Smith G, Schneider M, et al. Bias in meta-analysis detected by a simple, graphical test. Br Med J 1997;315:629-34 CrossRef Medline

20. Beare R, Chen J, Phan TG; VISTA-Acute Collaboration. Collaboration VI-A: googling stroke ASPECTS to determine disability-exploratory analysis from VISTA-Acute Collaboration. PLoS One 2015;10:e125687 CrossRef Medline

21. Fukuda K, Keppetipola K, Davison M, et al. Utility of aspects region location in predicting stroke thrombectomy outcomes. In: Proceedings of the Annual Meeting of the Society of NeuroInterventional Surgery, Colorado Springs, Colorado. July 24-27, 2017;9Suppl 10:A3-A4

22. Payabvash S, Benson JC, Tyan AE, et al. Multivariate prognostic model of acute stroke combining admission infarct location and symptom severity: a proof-of-concept study. J Stroke Cerebrovasc Dis 2018;27:936-44 CrossRef Medline

23. Haranhalli N, Mbabuike N, Grewal SS, et al. Topographic correlation of infarct area on CT perfusion with functional outcome in acute ischemic stroke. J Neurosurg 2019;132:33-41 CrossRef Medline

24. Rosso C, Blanc R, Ly J, et al; ASTER Trial and Pitié-Salpêtrière Investigators. Impact of infarct location on functional outcome following endovascular therapy for stroke. J Neurol Neurosurg Psychiatry 2019;90:313-19 CrossRef Medline

25. Arnold M, Slezak A, El-Koussy M, et al. Occlusion location of middle cerebral artery stroke and outcome after endovascular treatment. Eur Neurol 2015;74:315-21 CrossRef Medline
26. Cheng B, Forkert ND, Zavaglia M, et al. Influence of stroke infarct location on functional outcome measured by the modified Rankin scale. Stroke 2014;45:1695-1702 CrossRef Medline

27. Sheth KN, Terry JB, Nogueira RG, et al. Advanced modality imaging evaluation in acute ischemic stroke may lead to delayed endovascular reperfusion therapy without improvement in clinical outcomes. J Neurointerv Surg 2013;5(Suppl 1):i62-65 CrossRef Medline

28. McTaggart RA, Jovin TG, Lansberg MG, et al; DEFUSE 2 Investigators. Alberta stroke program early computed tomographic scoring performance in a series of patients undergoing computed tomography and MRI: reader agreement, modality agreement, and outcome prediction. Stroke 2015;46:407-12 CrossRef Medline

29. Payabvash S, Kamalian S, Fung S, et al. Predicting language improvement in acute stroke patients presenting with aphasia: a multivariate logistic model using location-weighted atlas-based analysis of admission CT perfusion scans. AJNR Am J Neuroradiol 2010;31:1661-68 CrossRef Medline

30. Payabvash S, Souza LC, Kamalian S, et al. Location-weighted CTP analysis predicts early motor improvement in stroke: a preliminary study. Neurology 2012;78:1853-59 CrossRef Medline

31. Almekhlafi MA, Hill MD, Roos YM, et al. Stroke laterality did not modify outcomes in the HERMES meta-analysis of individual patient data of 7 trials. Stroke 2019;50:2118-24 CrossRef Medline

32. Baek BH, Lee YY, Kim SK, et al. Pretreatment anterior choroidal artery infarction predicts poor outcome after thrombectomy in intracranial ICA occlusion. AJNR Am J Neuroradiol 2019;40:1349-55 CrossRef Medline

33. Tan BY, Kong WY, Ngiam JN, et al. The role of topographic collaterals in predicting functional outcome after thrombolysis in anterior circulation ischemic stroke. J Neuroimaging 2017;27:217-20 CrossRef Medline

34. Barber PA, Demchuk DM, Zhang J, et al, Validity and reliability of a quantitative CT score in predicting outcome of hyperacute stroke before thrombolytic therapy: ASPECTS Study Group. Alberta Stroke Programme Early CT Score. Lancet 2000;355:1670-74 CrossRef Medline 